\title{
Cerebrospinal Fluid Neurogranin as a Biomarker of Neurodegenerative Diseases: A Cross-Sectional Study
}

\author{
Simone Lista, $\mathrm{PhD}^{\mathrm{a}, \mathrm{b}}$; Nicola Toschi, $\mathrm{PhD}^{\mathrm{c}, \mathrm{d}, \mathrm{e}}$; Filippo Baldacci, $\mathrm{MD}^{\mathrm{f}, \mathrm{g}}$;
}

Henrik Zetterberg, $\mathrm{MD}^{\mathrm{h}, \mathrm{i}}$, PhD; Kaj Blennow, MD, $\mathrm{PhD}^{\mathrm{h}, \mathrm{j}}$; Ingo Kilimann, $\mathrm{MD}^{\mathrm{k}}$;

Stefan J. Teipel, MD ${ }^{\mathrm{k}}$; Enrica Cavedo, $\mathrm{PhD}^{\mathrm{a}, \mathrm{g}, \mathrm{l}}$; Antonio Melo dos Santos, MD ${ }^{\mathrm{g}}$;

Stéphane Epelbaum, MD ${ }^{\mathrm{g}}$; Foudil Lamari, $\mathrm{MD}^{\mathrm{m}}$; Bruno Dubois, $\mathrm{MD}^{\mathrm{g}}$; Robert Nisticò, $\mathrm{MD}^{\mathrm{n}}$;

Roberto Floris, $\mathrm{MD}^{\mathrm{c}}$; Francesco Garaci, $\mathrm{MD}^{\mathrm{c}, \mathrm{n}}$; and Harald Hampel, MD, $\mathrm{PhD}^{\mathrm{a}, \mathrm{g}}$

a AXA Research Fund \& UPMC Chair, Paris, France

${ }^{b}$ IHU-A-ICM - Paris Institute of Translational Neurosciences, Pitié-Salpêtrière University

Hospital, Paris, France

c Department of Biomedicine and Prevention, University of Rome "TorVergata", Rome, Italy

d Department of Radiology, Athinoula A. Martinos Center for Biomedical Imaging, Boston,

MA, USA

${ }^{\text {e }}$ Harvard Medical School, Boston, MA, USA

${ }^{f}$ Department of Clinical and Experimental Medicine, University of Pisa, Pisa, Italy

g Sorbonne Universities, Pierre and Marie Curie University, Paris 06, Institute of Memory and Alzheimer's Disease (IM2A) \& Brain and Spine Institute (ICM) UMR S 1127, Department of Neurology, Pitié-Salpêtrière University Hospital, Paris, France

h Institute of Neuroscience and Physiology, Department of Psychiatry and Neurochemistry,

The Sahlgrenska Academy at the University of Göteborg, Mölndal, Sweden

i Department of Molecular Neuroscience, UCL Institute of Neurology, Queen Square, London, UK

$\mathbf{j}$ The Torsten Söderberg Professorship in Medicine at the Royal Swedish Academy of Sciences, Sweden

${ }^{\mathbf{k}}$ Department of Psychosomatic Medicine, University of Rostock \& DZNE Rostock, Rostock, Germany

IIRCCS “San Giovanni di Dio-Fatebenefratelli”, Brescia, Italy,

m AP-HP, UF Biochimie des Maladies Neuro-métaboliques, Service de Biochimie

Métabolique, Groupe Hospitalier Pitié-Salpêtrière, Paris, France

"Casa di Cura "San Raffaele Cassino", Cassino, Italy

Manuscript requirements

Title character count

87 characters (max. 96 including spaces)

Number of references:

39 (max. 40)

Number of tables:

2

Number of figures:

Word count abstract

3

Word count paper

247 words $(\max .250)$

2,750 words $(\max . \mathbf{3 , 0 0 0})$

\section{Corresponding Author:}

Simone Lista, $\mathrm{PhD}$

AXA Research Fund \& UPMC Chair,

IHU-A-ICM - Paris Institute of Translational Neurosciences,

Pitié-Salpêtrière University Hospital,

47 Boulevard de l'Hôpital, 75651 - Paris, CEDEX 13, France.

Phone: +3315727 4674

Fax: +33142167516

E-Mail: slista@libero.it (S. Lista) 


\section{E-Mail addresses of all authors}

Simone Lista: slista@ libero.it

Nicola Toschi: toschi@med.uniroma2.it

Filippo Baldacci: filippo.baldacci@unipi.it

Henrik Zetterberg: henrik.zetterberg@ clinchem.gu.se

Kaj Blennow: kaj.blennow@neuro.gu.se

Ingo Kilimann: Ingo.Kilimann@dzne.de

Stefan J. Teipel: stefan.teipel@med.uni-rostock.de

Enrica Cavedo: enrica.cavedo@gmail.com

Antonio Melo dos Santos: neurologue.memoire@ gmail.com

Stéphane Epelbaum: stephane.epelbaum@aphp.fr

Foudil Lamari: foudil.lamari@ aphp.fr

Bruno Dubois: bruno.dubois@ aphp.fr

Robert Nisticò: robert.nistico@gmail.com

Roberto Floris: roberto.floris@ uniroma2.it

Francesco Garaci: garaci@gmail.com

Harald Hampel: Harald.Hampel@med.uni-muenchen.de

Statistical analysis conducted by Dr. Nicola Toschi, $\mathrm{PhD}$

Department of Biomedicine and Prevention, University of Rome "TorVergata", Rome, Italy; Department of Radiology, Athinoula A. Martinos Center for Biomedical Imaging, Boston, MA, USA; Harvard Medical School, Boston, MA, USA

E-Mail: toschi@med.uniroma2.it

Search Terms: [26] Alzheimer's disease, [29] Frontotemporal dementia, [36] Cognitive aging, [39] MCI (mild cognitive impairment), [319] Cerebrospinal Fluid

\section{Author Contributions:}

\section{Study Funding:}

Dr. H. Hampel is supported by the AXA Research Fund, the Fondation Universite Pierre et Marie Curie and the "Fondation pour la Recherche sur Alzheimer", Paris, France. The research leading to these results has received funding from the program "Investissements d'avenir" ANR10-IAIHU-06 (Agence Nationale de la Recherche-10-IA Agence Institut Hospitalo-Universitaire$6)$.

Dr. H. Zetterberg is a Wallenberg Academy Fellow. Dr. K. Blennow holds the Torsten Söderberg Professorship of Medicine.

\section{Disclosures:}

$\mathrm{KB}$ and $\mathrm{HZ}$ are co-founders of Brain Biomarker Solutions in Gothenburg AB, a GU Venturebased platform company at the University of Gothenburg. 


\section{ABSTRACT}

We investigated cerebrospinal fluid (CSF) concentrations of the postsynaptic biomarker neurogranin at baseline in cognitively healthy controls (HC) compared to individuals with mild cognitive impairment (MCI), patients with Alzheimer's disease (AD) dementia, and patients with frontotemporal dementia (FTD). CSF neurogranin was quantified using an in-house immunoassay in a cross-sectional multicenter study of 108 participants [AD dementia $(n=35)$, FTD $(n=9), \operatorname{MCI}(n=41)$, cognitively HC $(n=23)]$. CSF neurogranin concentrations were significantly higher in AD patients compared with both HC subjects and FTD patients, suggesting that increased CSF neurogranin concentrations may indicate AD-related pathophysiology. CSF neurogranin was independently associated with both total tau and hyperphosphorylated tau proteins, whereas a non-significant correlation with the 42 -amino acidlong amyloid- $\beta$ peptide was evident. CSF neurogranin, however, was not superior to core AD biomarkers in differentiating $\mathrm{HC}$ from the three diagnostic groups, and it did not improve their diagnostic accuracy. We conclude that further classification and longitudinal studies are required to shed more light into the potential role of neurogranin as a pathophysiological biomarker of neurodegenerative diseases.

\section{INTRODUCTION}

Synaptic pathology plays a critical role in Alzheimer's disease (AD) ${ }^{1,2}$ and correlates with cognitive decline.$^{3-6}$ Because "synapse failure" is increasingly recognized as a hallmark of AD, ${ }^{7}$ synaptic markers hold promise for the diagnosis and monitoring of this condition. Neurogranin a postsynaptic protein mainly localized into dendritic spines of neurons within associative cortical areas ${ }^{8-10}-$ is involved in synaptic plasticity. ${ }^{11}$ Neurogranin expression is significantly 
lower in the cortex and hippocampus of post-mortem $\mathrm{AD}$ brains versus controls. ${ }^{2,12}$ Compared with healthy controls (HCs), CSF neurogranin concentrations are increased in $\mathrm{AD}^{13}$ and mild cognitive impairment (MCI) converting into AD (MCI-AD). ${ }^{14-16}$ Moreover, neurogranin predicts MCI to AD dementia progression, ${ }^{14,17,18}$ and the rate of cognitive decline, ${ }^{14}$ and correlates longitudinally with hippocampal atrophy rates, ${ }^{17,19}$ as well as with future reduction in cortical glucose metabolism assessed by ${ }^{18}$ F-Fluorodeoxyglucose-PET $\left({ }^{18}\right.$ F-FDG-PET $) .{ }^{17}$

Herein, we investigated the diagnostic accuracy of CSF neurogranin in distinguishing clinical AD dementia patients from HCs and frontotemporal dementia (FTD) patients. Furthermore, we examined neurogranin diagnostic accuracy across the spectrum of AD pathology using an unbiased descriptive categorization system, the "A/T/N" scheme, based on core AD biomarkers and independent of cognitive impairment severity. ${ }^{20}$ It includes three biomarker categories reflecting $\mathrm{AD}$ pathophysiology, where " $\mathrm{A}$ " refers to amyloid-beta $(\mathrm{A} \beta)$ pathology, " $\mathrm{T}$ " to tau pathology, and "N" to neurodegeneration. To this end, we tested whether CSF neurogranin distinguished HCs from I) AD pathology patients (presenting decreased CSF concentrations of $A \beta_{1-42}$ peptide and increased amounts of total tau ( $t$-tau) or hyperphosphorylated tau ( $p$-tau)

protein), $\left.{ }^{21} \mathrm{II}\right)$ patients showing tau pathology only, and III) patients with $\mathrm{A} \beta$ pathology only. We also assessed the ability of neurogranin to discriminate AD pathology from FTD.

\section{MATERIALS AND METHODS}

\section{Standard protocol approvals, registrations, and patient consents}

The study was conducted in accordance with the tenets of the Declaration of Helsinki and was approved by the local Ethical Committees at each participant university. All participants or their representatives gave written informed consent for the use of their clinical data for research purposes. 


\section{Study participants}

The research was designed as a multicentre cross-sectional study retrospectively conducted in a convenience series from three independent European academic AD research centres and memory clinics. A total of 108 individuals were included. Specifically, 35 participants were recruited from the Institute of Memory and Alzheimer's Disease (Institut de la Mémoire et de la Maladie d'Alzheimer, IM2A) at Pitié-Salpêtrière University Hospital in Paris (France), 57 from the German Centre for Neurodegenerative Diseases (DZNE) in Rostock (Germany), and 16 from the Institute of Neuroscience and Physiology at Sahlgrenska University Hospital in Mölndal (Sweden).

\section{Patient stratification}

\section{Categorization according to the clinical diagnostic approach ("Level I")}

The clinical diagnosis of AD dementia was performed according to the National Institute of Neurological and Communicative Disorders and Stroke-Alzheimer's Disease and Related Disorders Association (NINCDS-ADRDA) consensus criteria. ${ }^{22}$ The clinical diagnosis of MCI was based on MCI core clinical criteria. ${ }^{23}$ The diagnosis of the FTD was performed according to the consensus on clinical diagnostic criteria of $1998 .{ }^{24} \mathrm{HCs}$ were individuals who I) volunteered for a lumbar puncture, II) showed a negative history of neurological or psychiatric diseases, and III) had a Mental-State Examination (MMSE) score between 27 and 30.

Of the 23 cognitively HCs (first group), two individuals from the Gothenburg cohort showed CSF t-tau concentrations higher than the established cut-off value. Being asymptomatic-at-risk of $\mathrm{AD}^{21}$ or preclinical $\mathrm{AD} .^{25}$ they were excluded from further analyses. The second group consisted of 41 clinically defined MCI cases. ${ }^{23}$ The third and fourth groups included 35 AD patients $^{21}$ and 9 FTD patients, respectively (Figure 1). ${ }^{24}$ 


\section{Categorization following the A/T/N system ("Level II")}

$\mathrm{AD}$ and MCI patient classification followed the biomarker-based descriptive stratification model (“A/T/N" system) recently proposed by Jack and colleagues. ${ }^{20}$ It contemplates 3 binary (positive/negative) categories: "A" referring to an amyloid biomarker (CSF A $\beta_{1-42}$ or amyloidPET), "T" to a tau pathology biomarker (CSF p-tau or tau-PET), and "N" to a quantitative or topographic biomarker of neurodegeneration or neuronal injury [CSF t-tau, ${ }^{18} \mathrm{~F}-\mathrm{FDG}-\mathrm{PET}$, or structural MRI]. Because each individual score is displayed with an " $\mathrm{A} \pm / \mathrm{T} \pm / \mathrm{N} \pm$ " arrangement, eight different categories are generated..$^{20}$ Notably, this stratification model is linked to the biomarker categorization frameworks - namely the International working group-2 (IWG-2) criteria $^{21}$ and the National Institute on Aging-Alzheimer's Association (NIA-AA) guidelines $^{22,23,25}$ - and is able to chart both diagnostic classification criteria. Here, we applied a simplified version of the original $\mathrm{A} / \mathrm{T} / \mathrm{N}$ model by including only core neurochemical markers (CSF A $\beta_{1-42,}$ t-tau, and p-tau). Overall, 5 categories were established as follows: Group 1, cognitively HCs $(\mathrm{n}=21)$, a priori defined as $[\mathrm{A}-/ \mathrm{T}-/ \mathrm{N}-]$, i.e. negative for all the core biological markers; Group 2, [A-/T-/N-] $(\mathrm{n}=15)$, including 2 patients diagnosed with AD dementia and 13 MCI patients negative to both $\mathrm{A} \beta$ and tau markers; Group 3, [A-/T $\pm / \mathrm{N}+, \mathrm{A}-/ \mathrm{T}+/ \mathrm{N} \pm](\mathrm{n}=15)$, including $6 \mathrm{AD}$ dementia patients and 9 MCI cases all positive to only tau markers; Group 4, [A+/T-/N-] $(\mathrm{n}=13)$, including 5 patients clinically diagnosed with AD dementia and comprising $8 \mathrm{MCI}$ cases displaying positivity to only $\mathrm{A} \beta$; Group 5, $[\mathrm{A}+/ \mathrm{T} \pm / \mathrm{N}+, \mathrm{A}+/ \mathrm{T}+/ \mathrm{N} \pm](\mathrm{n}=33)$, including $22 \mathrm{AD}$ dementia patients in line with the IWG-2 criteria ${ }^{21}$ and the NIA-AA guidelines ${ }^{22}$ comprising 11 prodromal $\mathrm{AD}^{26}$ or $\mathrm{MCI}$ due to $\mathrm{AD}$ cases, ${ }^{23}$ all showing positivity to both $A \beta$ and tau markers. Finally, the FTD participants $(n=9)($ Group 6) included seven patients who were both $A \beta_{1-42}$ and tau-negative, one patient who was $A \beta_{1-42}$ negative and tau positive,

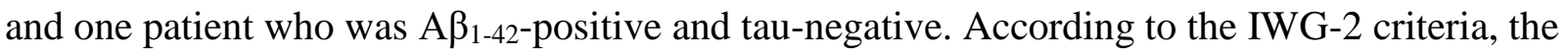
latter participant should be defined as a case of FTD and not as a patient with a frontal variant of 
AD. ${ }^{21}$ Of note, since the A/T/N system is not directly applicable to FTD, this last group (VI) was examined exclusively in terms of clinical diagnosis (Figure 1).

\section{CSF sampling}

A diagnostic lumbar puncture was performed in all participants. All CSF samples included in the three study cohorts were collected in polypropylene tubes, centrifuged at $1000 \mathrm{~g}$ for $10 \mathrm{~min}$ at $+4^{\circ} \mathrm{C}$ (samples collected at IM2A in Paris), $1500 \mathrm{~g}$ for $10 \mathrm{~min}$ at $+4^{\circ} \mathrm{C}$ (samples collected at DZNE in Rostock), $1800 \mathrm{~g}$ for $10 \mathrm{~min}$ at $+4^{\circ} \mathrm{C}$ (samples collected at Mölndal Clinical Neurochemistry Laboratory). The collected supernatant was aliquoted and stored at $-80^{\circ} \mathrm{C}$ pending biochemical analysis.

\section{Immunoassays for CSF core biomarkers}

For the Paris cohort, CSF analyses of $\mathrm{A} \beta_{1-42,}, \mathrm{t}$-tau, $\mathrm{p}$-tau were performed at the Laboratory of Biochemistry, Unit of Biochemistry of Neurometabolic diseases, Pitié-Salpêtrière University Hospital of Paris. For the Rostock cohort, CSF analyses were executed in two different units: the Institute of Clinical Chemistry and Laboratory Medicine, Rostock University Medical Centre, after 06/2012, and the Laboratory of Neurochemistry, Department of Neurology, Göttingen University Medical Centre, before 06/2012. For the Göteborg cohort, CSF analyses were executed at the Clinical Neurochemistry Laboratory at the Sahlgrenska University Hospital, Mölndal. CSF A $\beta_{1-42}$, t-tau, and tau phosphorylated at threonine 181 (p-tau 181 ) concentrations were measured using established sandwich ELISA methods, INNOTEST $\beta$-AMYLOID(1-42), ${ }^{27}$ INNOTEST hTAU-Ag, ${ }^{28}$ and INNOTEST Phospho-Tau[181P $]^{29}$ (Fujirebio Europe NV, Gent, Belgium), respectively. All analyses were performed by board-certified laboratory technicians blinded to clinical information. CSF biomarkers abnormalities were defined based on reference values currently utilized in each memory clinic: at IM2A in Paris, $A \beta_{1-42}<500 \mathrm{pg} / \mathrm{mL}$, t-tau $>450$ pg/mL, p-tau ${ }_{181}>60$ pg/mL; at DZNE in Rostock, $A \beta_{1-42}<567$ pg/mL, t-tau $>512$ pg/mL, p- 
$\operatorname{tau}_{181}>66 \mathrm{pg} / \mathrm{mL}$ for the CSF samples measured before $06 / 2012$ and $\mathrm{A} \beta_{1-42}<450 \mathrm{pg} / \mathrm{mL}$, t-tau $>450 \mathrm{pg} / \mathrm{mL}, \mathrm{p}-\operatorname{tau}_{181}>62 \mathrm{pg} / \mathrm{mL}$ for the CSF samples measured after 06/2012; at Mölndal Clinical Neurochemistry Laboratory, $\mathrm{A} \beta_{1-42}<550 \mathrm{pg} / \mathrm{mL}$, t-tau $>400 \mathrm{pg} / \mathrm{mL}, \mathrm{p}-\operatorname{tau}_{181}>80 \mathrm{pg} / \mathrm{mL}$.

\section{Immunoassay for CSF neurogranin}

All CSF neurogranin analyses were performed at the Clinical Neurochemistry Laboratory at the Sahlgrenska University Hospital, Mölndal, Sweden. We used the same methodology as the one employed in a previous study. ${ }^{14}$ In short, CSF neurogranin was measured using an in-house ELISA assay based on the monoclonal antibody $\mathrm{Ng} 7$ (epitope including amino acids $52-65$ on neurogranin) for capture, a polyclonal neurogranin anti-rabbit antibody (ab23570; Upstate Biotechnology, Lake Placid, NY, USA) for detection, and full-length neurogranin protein as calibrator. All analyses were performed on one occasion with randomized samples using one batch of reagents by board-certified laboratory technicians blinded to clinical information to avoid bias.

\section{Statistical Analysis}

The associations of diagnostic groups with sex and age were assessed with Fisher's exact tests and nonparametric Kruskal-Wallis (KW) tests, respectively. All neurogranin values were initially adjusted for age, sex, and site using nonparametric regression. This step allowed age-, sex-, and site- independent assessment of the diagnostic potential of neurogranin while foregoing assumptions of normality. Whenever the result of the KW test was statistically significant $(P<$ 0.05), post-hoc groupwise comparisons of neurogranin values were performed with the Conover's test for multiple comparisons. Results of post-hoc testing were corrected for multiple comparisons using a False Discovery Rate (FDR) procedure $(\alpha=0.05)$. We then evaluated the diagnostic potential of neurogranin using logistic regression within a Leave-One-Out CrossValidation (LOO-CV) approach in the following a priori comparisons: AD versus $\mathrm{HCs}, \mathrm{AD}$ 
versus $\mathrm{FTD}$, in Level I of categorization; $\mathrm{HCs}$ versus $[\mathrm{A}-/ \mathrm{T} \pm / \mathrm{N}+, \mathrm{A}-/ \mathrm{T}+/ \mathrm{N} \pm], \mathrm{HCs}$ versus $[\mathrm{A}+/ \mathrm{T}-/ \mathrm{N}-], \mathrm{HCs}$ versus $[\mathrm{A}+/ \mathrm{T} \pm / \mathrm{N}+, \mathrm{A}+/ \mathrm{T}+/ \mathrm{N} \pm]$, and $[\mathrm{A}+/ \mathrm{T} \pm / \mathrm{N}+, \mathrm{A}+/ \mathrm{T}+/ \mathrm{N} \pm]$ versus $\mathrm{FTD}$, in Level II. In this analysis, age-, sex-, and site-adjusted neurogranin values were entered as predictors and the diagnostic group was entered as the dependent variable. After model fitting, we calculated the area under the receiver operating characteristic (AUROC) curve by pooling predictions computed on the test sets from each train-test split in the LOO-CV procedure. Successively we computed its associated confidence intervals using a bootstrap procedure ${ }^{30}$ (100000 bootstraps). The discriminatory ability of neurogranin to correctly allocate participants to diagnostic groups was classified as follows: excellent (AUROC 0.90-1.00), good (AUROC 0.80-0.89), fair (AUROC 0.70-0.79), poor (AUROC 0.60-0.69), or fail/no discriminatory capacity (AUROC 0.50-0.59). ${ }^{31}$ All statistical analyses were performed in the R statistical environment version 3.2.3 (https://www.R-project.org/) under a Linux environment using the nonparametric kernel smoothing methods for mixed data types package (np package), ${ }^{32}$ partial ROC (pROC) package, ${ }^{30}$ and the pairwise multiple comparison of mean ranks (PMCMR) package. ${ }^{33}$ Two-tailed $P$ values $<0.05$ were considered statistically significant.

\section{RESULTS}

\section{CSF neurogranin concentrations (Level I)}

Table 1 summarizes the levels of all analytes, combined with demographic and clinical data of the population classified according to Level I. Compared with HCs, CSF neurogranin levels were significantly increased in AD patients $(P=0.004)$. Higher CSF neurogranin levels were observed in $\operatorname{AD}$ group $(P=0.004)$ compared with FTD group (Figure 2A).

\section{CSF neurogranin concentrations (Level II)}

Table 2 summarizes the levels of all analytes, combined with demographic and clinical data of the population classified according to Level II. Compared with HCs, CSF neurogranin levels were significantly increased in patients who were tau-positive only $[\mathrm{A}-/ \mathrm{T} \pm / \mathrm{N}+, \mathrm{A}-/ \mathrm{T}+/ \mathrm{N} \pm](P=$ 
$0.001)$ and in those positive to both $\mathrm{A} \beta$ and tau markers $[\mathrm{A}+/ \mathrm{T} \pm / \mathrm{N}+, \mathrm{A}+/ \mathrm{T}+/ \mathrm{N} \pm](P<0.001)$. These two groups also presented markedly higher CSF neurogranin concentrations compared with cases negative for all the core biomarkers $[\mathrm{A}-/ \mathrm{T}-/ \mathrm{N}-](P<0.001$ for both groups $)$, those who were A $\beta$-positive only $[\mathrm{A}+/ \mathrm{T}-/ \mathrm{N}-](P<0.001$ for both groups), and FTD patients $(P<$ 0.001 for both groups) (Figure 2B).

\section{Diagnostic accuracy of neurogranin (Level I)}

We found that CSF neurogranin was able to differentiate HCs from AD dementia patients with an AUROC of 0.72 (95\% CI, 0.58-0.86) (Figure 3A). CSF neurogranin was also able to discriminate AD from FTD with an AUROC of 0.76 (95\% CI, 0.55-0.96) (Figure 3B).

\section{Diagnostic accuracy of neurogranin (Level II)}

We found that CSF neurogranin was able to differentiate cognitively HCs from Group 3 [A$/ \mathrm{T} \pm / \mathrm{N}+, \mathrm{A}-/ \mathrm{T}+/ \mathrm{N} \pm]$ with an AUROC of 0.77 (95\% CI, 0.60-0.94) (Figure 3C). CSF neurogranin was able to discriminate HCs from Group 4 [A+/T-/N-] with an AUROC of 0.55 (95\% CI, 0.340.76) (Figure 3D). CSF neurogranin was able to distinguish $\mathrm{HCs}$ from Group $5[\mathrm{~A}+/ \mathrm{T} \pm / \mathrm{N}+$, $\mathrm{A}+/ \mathrm{T}+/ \mathrm{N} \pm]$ with an AUROC of 0.85 (95\% CI, 0.74-0.95) (Figure 3E). CSF neurogranin was able to discern Group $5[\mathrm{~A}+/ \mathrm{T} \pm / \mathrm{N}+, \mathrm{A}+/ \mathrm{T}+/ \mathrm{N} \pm]$ from FTD with an AUROC of $0.85(95 \% \mathrm{CI}$, 0.64-1.00) (Figure 3F).

\section{DISCUSSION}

Results of Level I (Figure 2A) are consistent with previous reports showing increased CSF neurogranin levels in AD dementia patients versus HCs. ${ }^{14-19,34,35}$. Increased CSF neurogranin concentrations in $\mathrm{AD}$ dementia are most likely caused by the extracellular release of synaptic proteins resulting from synaptic dysfunction and loss. Interestingly, higher CSF neurogranin concentrations were found in $\mathrm{AD}$ dementia versus $\mathrm{FTD}$, suggesting a potential role for 
neurogranin in discriminating between the two forms of dementia, in line with a prior study. ${ }^{34}$ To corroborate the increase of CSF neurogranin as an AD-specific characteristic, ${ }^{35}$ future analyses are needed across a range of different neurodegenerative diseases.

In Level II, the significantly elevated CSF neurogranin expression detected in all patient categories displaying tau pathology (Figure 2B) confirms the previously observed association between neurogranin and markers of neuronal injury. ${ }^{13,14,16-18,35}$ Increased amounts of CSF t-tau and other neuronal proteins in $\mathrm{AD}$ reflect extensive cortical neuro-axonal degeneration. Owing to the correlation between CSF neurogranin and tau levels, higher neurogranin concentrations may serve as a proxy for the cortical synaptic degeneration occurring in $\mathrm{AD} .^{2,36}$

ROC curves, computed in a LOO-CV setting, were used to examine the accuracy of neurogranin as an AD diagnostic marker. In Level I, the performances of CSF neurogranin in discriminating clinical AD dementia from HCs and FTD were both fair (AUROC 0.72 and 0.76, respectively) (Figures 3A and 3B). In Level II, the performances in distinguishing patients with $\mathrm{AD}$ pathology (i.e. exhibiting positivity to both $\mathrm{A} \beta$ and tau markers) from $\mathrm{HCs}$ as well as from FTD were good (AUROC 0.85 for both comparisons) (Figures 3E and 3F). Consequently, neurogranin delivers a higher diagnostic accuracy when the A/T/N classification system (rather than clinical criteria) is used. A fair AUROC (0.77) was found for tau positive patients versus HCs (Figure 3C), whereas the ability to discriminate between patients who were A $\beta$-positive only and HCs was unsatisfactory (AUROC 0.55) (Figure 3D). These results suggest that CSF neurogranin concentrations may reflect mostly tau pathology rather than $\mathrm{A} \beta$ pathology. ${ }^{14,18,35}$ Differently from the case of tau markers, the link between CSF A $\beta$ and neurogranin appears relatively weak and the association between amyloid plaque load and synaptic depletion appears very low if not absent at all. ${ }^{14,18,35}$ Accordingly, $A \beta$ is involved in the initial steps of $A D$ pathophysiology rather than in synaptic loss. ${ }^{37}$

Some potential caveats of this study merit comment. Owing to the relatively small sample size, it was not possible to divide our patients in all groups established by the original A/T/N 
system when Level II classification was used. ${ }^{20}$ Because only core CSF biomarkers (and not imaging modalities) were used, MCI with AD dementia patients were grouped together for the purpose of analysis (Figure 1). Notably, the clinical distinction between MCI and dementia might be problematic and time-dependent; accordingly, the IWG-2 criteria define MCI with AD pathology as $\mathrm{AD}$ in its prodromal stage. ${ }^{21}$ Owing to the cross-sectional nature of the study, it was not possible to differentiate stable-MCI cases from those converting into dementia. Further studies are needed to confirm the potential value of neurogranin in predicting MCI to AD conversion. ${ }^{17,18}$ Extensive psychometric data were not available in our study, preventing the study of CSF neurogranin levels in relation to different cognitive dimensions. Moreover, the quantification of core AD CSF biomarkers was not performed in a centralized manner, potentially being subject to inter- and intra-operator variability. This study is largely exploratory as it represents the first attempt of utilizing neurogranin as a CSF biomarker for AD diagnosis in a clinical setting by applying an original, unbiased biomarker-based model of stratification ${ }^{20}$ ) (Figure 1). Notably, the $\mathrm{A} / \mathrm{T} / \mathrm{N}$ dissection system addresses the need for a unifying conceptual approach to biomarkers employed in AD research. In fact, given its substantially unbiased descriptive nature which eludes disease labels, the $\mathrm{A} / \mathrm{T} / \mathrm{N}$ scheme could potentially be employed in any framework of existing and upcoming diagnostic criteria. Moreover, it could be, at some point, expanded to integrate key biomarkers of other relevant proteinopathies, genetic or epigenetic factors, ${ }^{38}$ and indicators of other pathologies such as cerebrovascular diseases or white matter pathologies which appear to impair cognitive function. ${ }^{39}$ Finally, the multicenter design of the study may introduce variation + a comment on how we dealt with this (if we did; please feel free to disregard this comment if it is hard to meet it...).

In conclusion, our cross-sectional study confirms and expands previous findings on the role of CSF neurogranin as a biomarker that consistently distinguishes both AD dementia patients from HCs. Because neurogranin helps discriminate AD from FTD, its increased CSF concentration seems to be AD-specific. Furthermore, the A/T/N system allows improving 
neurogranin diagnostic accuracy in distinguishing cognitively impaired patients with AD pathology and, to a lesser degree, tau pathology from HCs as well as AD pathology from FTD.

Future studies are needed to investigate whether CSF neurogranin may predict conversion to dementia in subjects at risk of developing AD.

\section{REFERENCES}

1. Scheff SW, Neltner JH, Nelson PT. Is synaptic loss a unique hallmark of Alzheimer's disease? Biochem Pharmacol 2014;88:517-528.

2. Davidsson P, Blennow K. Neurochemical dissection of synaptic pathology in Alzheimer's disease. Int Psychogeriatr 1998;10:11-23.

3. Scheff SW, Price DA, Schmitt FA, DeKosky ST, Mufson EJ. Synaptic alterations in CA1 in mild Alzheimer disease and mild cognitive impairment. Neurology 2007;68:1501-1508.

4. Blennow K, Bogdanovic N, Alafuzoff I, Ekman R, Davidsson P. Synaptic pathology in Alzheimer's disease: relation to severity of dementia, but not to senile plaques, neurofibrillary tangles, or the ApoE4 allele. J Neural Transm (Vienna) 1996;103:603-618.

5. Terry RD, Masliah E, Salmon DP, et al. Physical basis of cognitive alterations in Alzheimer's disease: Synapse loss is the major correlate of cognitive impairment. Ann Neurol 1991;30:572-580.

6. DeKosky S, Scheff S. Synapse loss in frontal cortex biopsies in Alzheimer's disease: Correlation with cognitive severity. Ann Neurol 1990;27:457-464.

7. Hardy J. The amyloid hypothesis for Alzheimer's disease: a critical reappraisal. J Neurochem 2009;110:1129-1134.

8. Díez-Guerra F. Neurogranin, a link between calcium/calmodulin and protein kinase $\mathrm{C}$ signaling in synaptic plasticity. IUBMB Life 2010;62:597-606.

9.Bogdanovic N, Davidsson P, Gottfries J, Volkman I, Winblad B, Blennow K. Regional and cellular distribution of synaptic proteins in the normal human brain. Brain Aging 2002;2:18-30.

10. Represa A, Deloulme JC, Sensenbrenner M, Ben-Ari Y, Baudier J. Neurogranin: immunocytochemical localization of a brain-specific protein kinase $\mathrm{C}$ substrate. J Neurosci 1990;10:3782-3792.

11. Zhong L, Cherry T, Bies CE, Florence MA, Gerges NZ. Neurogranin enhances synaptic strength through its interaction with calmodulin. EMBO J 2009;28:3027-3039. 
12. Reddy PH, Mani G, Park BS, et al. Differential loss of synaptic proteins in Alzheimer's disease: implications for synaptic dysfunction. J Alzheimers Dis 2005;7:103-117.

13. Thorsell A, Bjerke M, Gobom J, et al. Neurogranin in cerebrospinal fluid as a marker of synaptic degeneration in Alzheimer's disease. Brain Res 2010;1362:13-22.

14. Kvartsberg H, Duits FH, Ingelsson M, et al. Cerebrospinal fluid levels of the synaptic protein neurogranin correlates with cognitive decline in prodromal Alzheimer's disease. Alzheimers Dement 2015;11:1180-1190.

15. Kvartsberg H, Portelius E, Andreasson U, et al. Characterization of the postsynaptic protein neurogranin in paired cerebrospinal fluid and plasma samples from Alzheimer's disease patients and healthy controls. Alzheimers Res Ther 2015;7:40.

16. De Vos A, Jacobs D, Struyfs H, et al. C-terminal neurogranin is increased in cerebrospinal fluid but unchanged in plasma in Alzheimer's disease. Alzheimers Dement 2015;11:1461-1469.

17. Portelius E, Zetterberg H, Skillbäck T, et al. Cerebrospinal fluid neurogranin: relation to cognition and neurodegeneration in Alzheimer's disease. Brain 2015;138:3373-3385.

18. Kester MI, Teunissen CE, Crimmins DL, et al. Neurogranin as a Cerebrospinal Fluid Biomarker for Synaptic Loss in Symptomatic Alzheimer Disease. JAMA Neurol 2015;72:12751280.

19. Tarawneh R, D'Angelo G, Crimmins D, et al. Diagnostic and Prognostic Utility of the Synaptic Marker Neurogranin in Alzheimer Disease. JAMA Neurol 2016;73:561-571.

20. Jack CR Jr, Bennett DA, Blennow K, et al. A/T/N: an unbiased descriptive classification scheme for Alzheimer's disease biomarkers. Neurology Epub 2016 Jul 1. pii: 10.1212/WNL.0000000000002923.

21. Dubois B, Feldman HH, Jacova C, et al. Advancing research diagnostic criteria for Alzheimer's disease: the IWG-2 criteria. Lancet Neurol 2014;13:614-629.

22. McKhann GM, Knopman DS, Chertkow H, et al. The diagnosis of dementia due to Alzheimer's disease: recommendations from the National Institute on Aging-Alzheimer's Association workgroups on diagnostic guidelines for Alzheimer's disease. Alzheimers Dement 2011;7:263-269.

23. Albert MS, DeKosky ST, Dickson D, et al. The diagnosis of mild cognitive impairment due to Alzheimer's disease: recommendations from the National Institute on Aging-Alzheimer's Association workgroups on diagnostic guidelines for Alzheimer's disease. Alzheimers Dement. 2011;7:270-279.

24. Neary D, Snowden JS, Gustafson L, et al. Frontotemporal lobar degeneration: a consensus on clinical diagnostic criteria. Neurology 1998;51:1546-1554.

25. Sperling RA, Aisen PS, Beckett LA, et al. Toward defining the preclinical stages of Alzheimer's disease: recommendations from the National Institute on Aging-Alzheimer's 
Association workgroups on diagnostic guidelines for Alzheimer's disease. Alzheimers Dement 2011;7:280-292.

26. Dubois B, Feldman HH, Jacova C, et al. Revising the definition of Alzheimer's disease: a new lexicon. Lancet Neurol 2010;9:1118-1127.

27. Vanderstichele H, Van Kerschaver E, Hesse C, et al. Standardization of measurement of beta-amyloid(1-42) in cerebrospinal fluid and plasma. Amyloid 2000;7:245-258.

28. Blennow K, Wallin A, Ågren H, Spenger C, Siegfried J, Vanmechelen E. Tau protein in cerebrospinal fluid: a biochemical diagnostic marker for axonal degeneration in Alzheimer's disease? Mol Chem Neuropathol 1995;26:231-245.

29. Vanmechelen E, Vanderstichele H, Davidsson P, et al. Quantification of tau phosphorylated at threonine 181 in human cerebrospinal fluid: a sandwich ELISA with a synthetic phosphopeptide for standardization. Neurosci Lett 2000;285:49-52.

30. Robin X, Turck N, Hainard A, et al. pROC: an open-source package for R and S+ to analyze and compare ROC curves. BMC Bioinformatics. 2011;12:77.

31. Xia J, Broadhurst DI, Wilson M, Wishart DS. Translational biomarker discovery in clinical metabolomics: an introductory tutorial. Metabolomics 2013;9:280-299.

32. Hayfield T, Racine JS. Nonparametric Econometrics: The np Package. Journal of Statistical Software 2008;27:1-32. Available at: https://www.jstatsoft.org/article/view/v027i05. Accessed July 25, 2016.

33. Pohlert T. The Pairwise Multiple Comparison of Mean Ranks Package (PMCMR). 2014. Available at: https://cran.r-project.org/web/packages/PMCMR/vignettes/PMCMR.pdf. Accessed July 25, 2016.

34. Janelidze S, Hertze J, Zetterberg H, et al. Cerebrospinal fluid neurogranin and YKL-40 as biomarkers of Alzheimer's disease. Ann Clin Transl Neurol 2015;3:12-20.

35. Wellington H, Paterson RW, Portelius E, et al. Increased CSF neurogranin concentration is specific to Alzheimer disease. Neurology 2016;86:829-835.

36. Ingelsson M, Fukumoto H, Newell KL, et al. Early A $\beta$ accumulation and progressive synaptic loss, gliosis, and tangle formation in AD brain. Neurology 2004;62:925-931.

37. Spires-Jones TL, Hyman BT. The intersection of amyloid beta and tau at synapses in Alzheimer's disease. Neuron 2014;82:756-771.

38. Lista S, Garaci FG, Toschi N, Hampel H. Imaging epigenetics in Alzheimer's disease. Curr Pharm Des 2013;19:6393-6415.

39. Mascalchi M, Ginestroni A, Toschi N, et al. The burden of microstructural damage modulates cortical activation in elderly subjects with MCI and leuko-araiosis. A DTI and fMRI study. Hum Brain Mapp 2014;35:819-830. 